\title{
Application of gel electrolyte in dye sensitized solar cells
}

\author{
P. Nijisha, N. M. Bhabhina, S. Sindhu* \\ Department of Nanoscience and Technology, University of Calicut, Kerala-673635, India \\ nijisha31@gmail.com, bhabhinanm@gmail.com, *sindhu.swaminath@gmail.com
}

PACS 88.40.fh, 88.40.H, 88.40.hj, 88.40.J

DOI 10.17586/2220-8054-2016-7-4-752-754

\begin{abstract}
The volatility of liquid electrolytes has been a major problem for their application in dye-sensitized solar cells (DSSC). In this study, liquid electrolyte was replaced by polymer based gel electrolyte. Polyvinyl alcohol is chosen as the polymeric matrix to gelate the liquid electrolyte with iodide-triiodide redox couple and suitable organic solvent. The amorphous nature and the physical cross linking formed between polymerpolymer and polymer-solvent is analyzed from XRD and FT-IR. Cell was fabricated and characterization was done. I-V and EIS measurements of the cell was taken. Easy fabrication and its advantages over liquid electrolyte makes gel electrolyte a promising alternative for liquid electrolyte.
\end{abstract}

Keywords: dye sensitized solar cells, quasi solid state dye sensitized solar cells, gel electrolyte.

Received: 14 February 2016. Revised: 26 April 2016.

\section{Introduction}

DSSC have received great attention owing to their low production costs and high efficiencies [1,2]. The certified record efficiency of DSSC is $12-13 \%$. A DSSC consists of a photo anode, a semiconductor film coated on a transparent conducting oxide layer, which is dye sensitized, a counter electrode, usually FTO coated with a catalytic material and electrolyte which includes a redox couple usually $\mathrm{I}^{-} / \mathrm{I}_{3}^{-}$system and a suitable solvent. In DSSC, the electrolyte is the medium for charge transportation between the two electrodes and acts as a source for dye regeneration [3]. The long term stability of the device strongly depends on the electrolyte component [4]. The highest efficiency reported in DSSC was by employing liquid electrolyte. But there are some practical impediments when using this liquid electrolyte like leakage while sealing and volatilization of the solvent, desorption and photodegradation of the attached dye, corrosion of the counter electrode etc., which prevent DSSC's from further application and commercialization $[4,5]$. So, as an alternative to liquid electrolyte, solid state electrolytes and quasi solid state electrolytes (gel electrolytes) were introduced. Though solid state electrolytes seem to be an ideal material for DSSC, the efficiency was not up to expectations [6]. This might be due to poor interfacial contact and poor charge carrier mobility. When coming to gel electrolyte, all the problems related to liquid and solid state electrolyte can be resolved. It remains as quasi solid at room temperature and as a highly viscous liquid at high temperatures. Thus, a gel electrolyte possesses both the cohesive property of solid as well as diffusive transport property of liquid [7]. In the present work a polymer-based gel electrolyte is synthesized and its behavior in the performance of dye sensitized solar cell is studied by using two different photoanode materials $-\mathrm{TiO}_{2}$ and $\mathrm{ZnO}$.

\section{Experimental}

\subsection{Materials}

Fluorine doped tin oxide (FTO-7 //Sq.), N719 (Ru(dcbpy $\left.\left.)_{2}(\mathrm{NCS})_{2}\right)-95 \%\right)$, Hexachloroplatinic acid $\left(\mathrm{H}_{2} \mathrm{PtCl}_{6}\right)$, Potassium iodide (KI) (bio ultra, $99.5 \%$ ), Polyvinyl alcohol (PVA, $99 \%$ hydrolyzed) were purchased from SigmaAldrich. Dimethyl sulfoxide (DMSO) and Iodine $\left(\mathrm{I}_{2}\right)$ is supplied by MERCK.

\subsection{Gel electrolyte synthesis}

Polyvinyl alcohol is used as the polymeric matrix. Gel electrolyte is prepared by adding adequate amount of polymer, $\mathrm{KI}$ and $\mathrm{I}_{2}$ to dimethyl sulfoxide. The resulting mixture is heated to $2 \mathrm{hrs}$ and stirred continuously to obtain the gel. 


\subsection{Assembling of quasi solid state dye sensitized solar cell}

The nanocrystalline $\mathrm{TiO}_{2}$ photo anode was fabricated by doctor blading $\mathrm{TiO}_{2}$ paste on FTO substrate. The film was air dried and sintered at $450^{\circ} \mathrm{C}$ for 30 mins. The sintered sample was then cooled to room temperature and was dipped in the dye solution for $24 \mathrm{hrs}$. The dye loaded sample was then air dried and sealed. The counter electrode was made by electrodepositing Pt on FTO surface. The two electrodes were then kept face to face with gel electrolyte in between to obtain a sandwich structure and is then clamped tightly.

\section{Results and discussion}

\subsection{Gel characterization}

Figure 1 shows the FT-IR of pure PVA and PVA based gel electrolyte. The presence of broad band at 3416 shows the inter-molecular hydrogen bonding. In PVA-G, the intensity of the band was found to increase, and this is because of extensive hydrogen bonding between the polymeric chains due to its complete expansion. The band at $2914 \mathrm{~cm}^{-1}$ shows the $-\mathrm{CH}$ - stretching vibration. The band at $1654 \mathrm{~cm}^{-1}$ is for terminal vinyl group. Bands at $1783 \mathrm{~cm}^{-1}$ and $1806 \mathrm{~cm}^{-1}$ correspond to the ester group present. The $1315 \mathrm{~cm}^{-1}$ and $1434 \mathrm{~cm}^{-1}$ bands correspond to S-O stretching, while those at $1024 \mathrm{~cm}^{-1}, 1083 \mathrm{~cm}^{-1}, 1186 \mathrm{~cm}^{-1}$ show the C-O vibration of PVA.

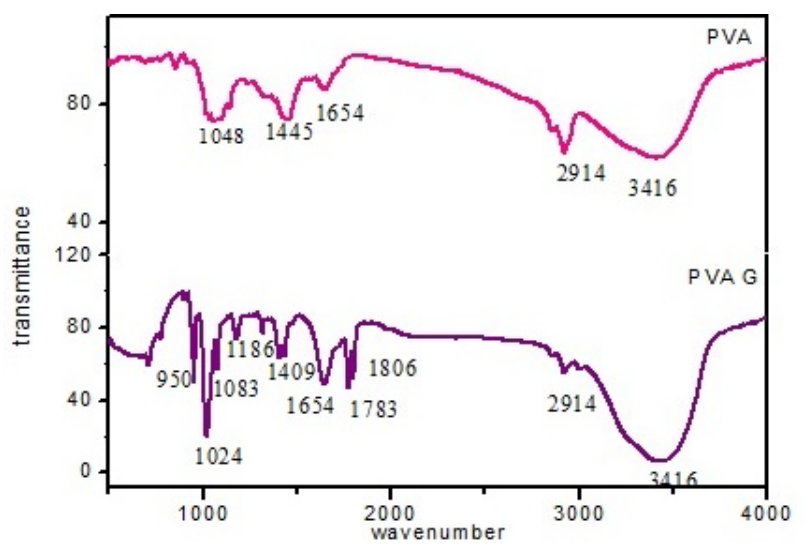

FIG. 1. FT-IR spectra of pure PVA and PVA gel electrolyte

The XRD spectra of gel electrolyte, pure PVA, KI, and $\mathrm{I}_{2}$ are shown in figure 2. Diffraction peak at $2 \theta-$ 40.76 is ascribed to pure PVA. Diffraction peak at $2 \theta-21.67,30.80,54.98$ and $24.62,25.07,29.04$ is for pure KI and $\mathrm{I}_{2}$ respectively, shows the crystalline nature of the ionic salt. An amorphous peak is observed for polymer gel electrolyte, which shows the complete dissolution of the ionic salt and also increased liquid electrolyte uptake by the matrix.

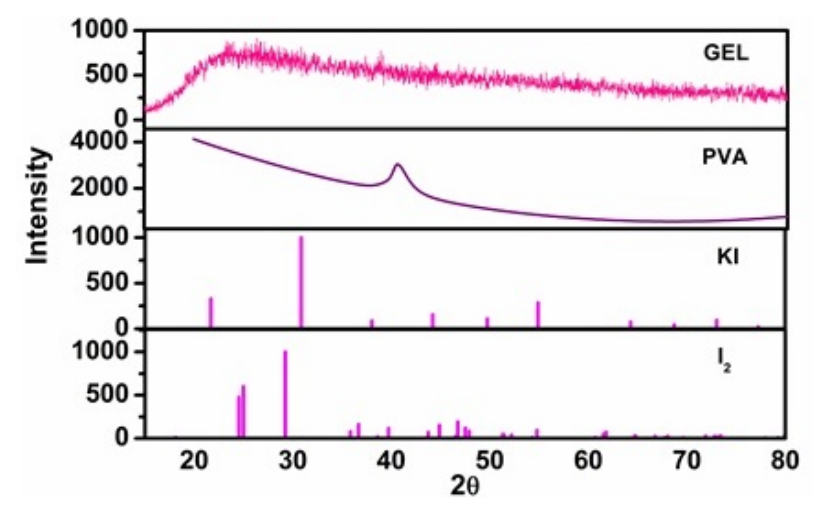

FIG. 2. XRD of pure $\mathrm{I}_{2}, \mathrm{KI}, \mathrm{PVA}$ and polymer gel electrolyte 


\subsection{Cell characterization}

The current-voltage characteristics and Nyquist plot of the DSSCs based on polymer gel electrolyte were performed with two different photoanode semiconductor materials- $\mathrm{TiO}_{2}$ and $\mathrm{ZnO}$. The table below summarizes the best values of their photovoltaic parameters.
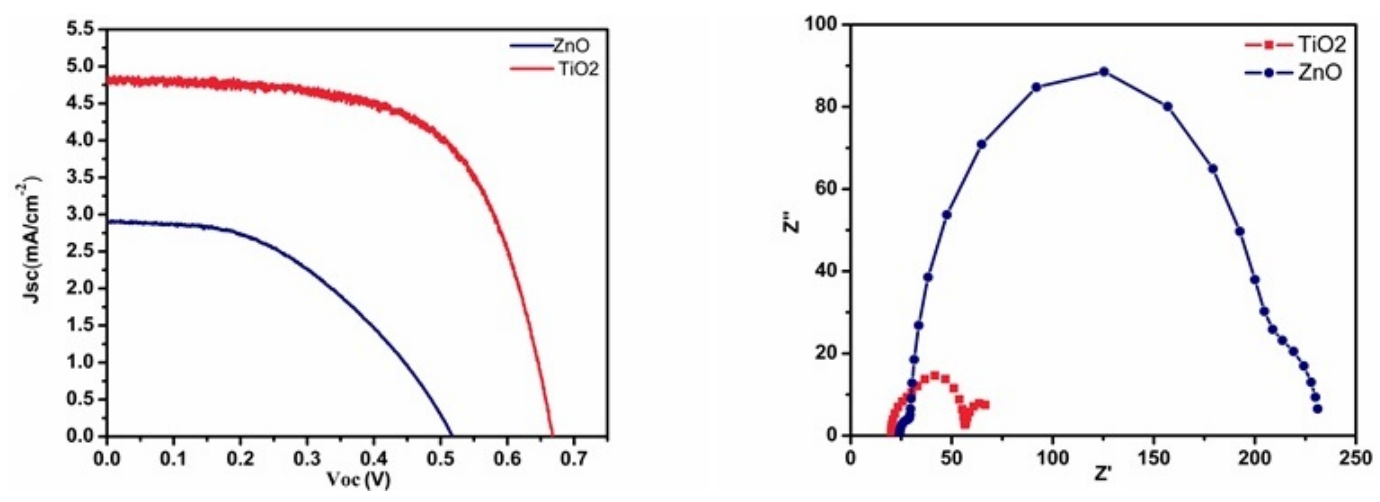

FIG. 3. (a) Jsc-Voc curve, (b) nyquist plot of the cell

TABLE 1. Photovoltaic parameters of $\mathrm{TiO}_{2}$ and $\mathrm{ZnO}$ based DSCs with gel electrolyte

\begin{tabular}{|c|c|c|c|c|}
\hline Photoanode material & $\mathbf{J s c}\left(\mathbf{m A} / \mathbf{c m}^{2}\right)$ & $\mathbf{V o c}(\mathbf{V})$ & $\mathbf{F F}(\%)$ & $\mathbf{E f f ( \% )}$ \\
\hline $\mathrm{TiO}_{2}$ & 1.21 & 0.67 & 63.6 & 2.05 \\
\hline $\mathrm{ZnO}$ & 0.723 & 0.517 & 45.8 & 0.684 \\
\hline
\end{tabular}

The obtained efficiencies are 2.05 and $0.68 \%$ respectively for $\mathrm{TiO}_{2}$ and $\mathrm{ZnO}$ based DSSC with PVA gel electrolyte. Here, the pure PVA based gel electrolyte of $\mathrm{KI} / \mathrm{I}_{2}$ in $\mathrm{DMSO} / \mathrm{EC}$ mixture without ionic liquid and any additives or fillers is used. An enhancement in the efficiency is anticipated with ionic liquid and other additives in the gel electrolyte and more studies in this direction are ongoing to enhance the efficiency. The efficiency values are low compared to perovskite-based DSSC. Perovskites give better efficiency and absorption coefficient as a sensitizer along with conventional electrolytes. However, focus of current work is on the synthesis of gel electrolyte, a better substitute for liquid electrolytes, and its application in electrochemical cells.

The charge transfer or transport behavior of the cell was measured by utilizing electrochemical impedance spectroscopy. The three semicircle from left to right gives the electrochemical behavior at the $\mathrm{Pt}$ counter electrode, $\mathrm{TiO}_{2} /$ dye/electrolyte interface and Warburg diffusion. The resistance offered at each interface when using $\mathrm{ZnO}$ as photoanode is very high when compared to the cell fabricated with $\mathrm{TiO}_{2}$ as the photoanode. This confirms the lower performance of the ZnO-based quasi solid state dye sensitized solar cell.

\section{Conclusion}

PVA based polymer gel electrolyte was synthesized. DSSC were fabricated with two different photoanode materials to study the performance of the cell. Photovoltaic studies show that the gel works well with $\mathrm{TiO}_{2}$-based solar cell, displaying an overall efficiency of $2.05 \%$.

\section{References}

[1] O'Regan B., Gratzel M. A low-cost, high-efficiency solar cell based on dye-sensitized colloidal $\mathrm{TiO}_{2}$ films. Nature, 1991, 353, P. 737-740.

[2] Yuh-Lang Lee., Yu-Jen Shen., Yu Min Yang. A hybrid PVDF-HFP/nanoparticle gel electrolyte for dye-sensitized solar cell application. Nanotechnology, 2008, 19, P. 455201.

[3] Nogueira A.F., Longo C., De Paoli M.A. Polymers in dye sensitized solar cells: overview and perspectives. Coordination Chemistry Reviews, 2004, 248, P. 1455-1468.

[4] Jo-Lin Lan, Tzu-Chien Wei, et al. Effects of iodine content in the electrolyte on the charge transfer and power conversion efficiency of dye sensitized solar cells under low light intensities. The J. Phys. Chem. C, 2012, 116, P. 25727-25733.

[5] Kun Seok Lee, Yongseok Jun, Jong Hyeok Park. Controlled Dissolution Of Polystyrene nanobeads: Transition from Liquid Electrolyte to Gel Electrolyte. Nano Lett., 2012, 12, P. 2233-2237.

[6] Chih-Hung Tsai, Chun-Yang Lu, et al. Efficient gel-state dye-sensitized solar cells adopting polymer gel electrolyte based on poly(methyl methacrylate). Organic electronics, 2013, 14, P. 3131-3137.

[7] Wataru Kubo, Kei Murakoshi, et al. Quasi-solid-state dye-sensitized $\mathrm{TiO}_{2}$ solar cells:Effective charge transport in mesoporous space filled with gel electrolytes containing iodide and iodine. J.Phys.Chem. B, 2001, 105, P. 12809-12815. 